\title{
EXHAUSTIVE OPERATORS AND VECTOR MEASURES
}

\author{
by N. J. KALTON \\ (Received 28th January 1974)
}

\section{Introduction}

Let $S$ be a compact Hausdorff space and let $\Phi: C(S) \rightarrow E$ be a linear operator defined on the space of real-valued continuous functions on $S$ and taking values in a (real) topological vector space $E$. Then $\Phi$ is called exhaustive (7) if given any sequence of functions $f_{n} \in C(S)$ such that $f_{n} \geqq 0$ and

$$
\sup _{s} \sum_{n=1}^{\infty} f_{n}(s)<\infty
$$

then $\Phi\left(f_{n}\right) \rightarrow 0$. If $E$ is complete then it was shown in (7) that exhaustive maps are precisely those which possess regular integral extensions to the space of bounded Borel functions on $S$; this is equivalent to possessing a representation

$$
\Phi(f)=\int_{S} f(s) d \mu(s)
$$

where $\mu$ is a regular countably additive $E$-valued measure defined on the $\sigma$ algebra of Borel subsets of $S$.

In this paper we seek conditions on $E$ such that every continuous operator $\Phi: C(S) \rightarrow E$ (for the norm topology on $C(S)$ ) is exhaustive. If $E$ is a Banach space then Pelczynski (14) has shown that every exhaustive map is weakly compact; then we have from results in (2) and (16);

Theorem 1.1. If $E$ is a Banach space containing no copy of $c_{0}$, then every bounded $\Phi: C(S) \rightarrow E$ is exhaustive.

Theorem 1.2. If $E$ is a Banach space containing no copy of $l_{\infty}$, then if $S$ is $\sigma$-Stonian, every bounded $\Phi: C(S) \rightarrow E$ is exhaustive.

These results extend naturally to locally convex spaces, but here we study the general non-locally convex case. We show that Theorem 1.1 does indeed extend to arbitrary topological vector spaces; it seems likely that Theorem 1.2 extends also, but we here only prove special cases. In particular we prove Theorem 1.2 when $E$ is separable (generalising a result due originally to Grothendieck (6)).

\section{Operators on $c_{0}$}

We denote by $\left(e_{n}\right)$ the unit vector basis of $c_{0}$. If $M \subset \mathbb{N}$ is an infinite subset, then $c_{0}(M)$ is the subspace of $c_{0}$ of all sequences vanishing outside $M$. Let $c_{00}$ represent the subspace of all sequences which are eventually zero, and let

$$
A_{n}=\left\{t \in c_{00}:\|t\|_{\infty} \leqq 1 \quad t_{1}=t_{2}=\ldots=t_{n-1}=0\right\} . \quad(n \geqq 2)
$$


Now let $\Phi: c_{0} \rightarrow(E, \tau)$ be a continuous linear operator mapping $c_{0}$ into a metrisable topological vector space $(E, \tau)$. Let $\left(U_{n}\right)$ be a base of closed balanced $\tau$-neighbourhoods of 0 satisfying $U_{n+1}+U_{n+1} \subset U_{n}$ for $n \geqq 1$. Define

$$
V_{n}=\bigcap_{m=2}^{\infty}\left(U_{n}+\Phi\left(A_{m}\right)\right) \text {. }
$$

Lemma 2.1. $\left(V_{n}\right)$ is a base for a metrisable vector topology $\gamma(\Phi)$ on $E$.

Proof. Each $V_{n}$ is balanced since each $U_{n}$ and $\Phi\left(A_{m}\right)$ is balanced. Since $U_{n}$ is absorbent, $V_{n}$ is absorbent. In view of Köthe (10, p. 146), it is necessary only to show that $V_{n+1}+V_{n+1} \subset V_{n}$ for every $n$, in order to prove that $\left(V_{n}\right)$ defines a vector topology.

Suppose $x, y \in V_{n+1}$; then for any $m$

$$
x=u_{1}+\Phi(t)
$$

where $u_{1} \in U_{n+1}$ and $t \in A_{m}$. Since $t \in c_{00}$, there exists $p$ such that $t_{i}=0$ for $i \geqq p$. Then

where $t^{\prime} \in A_{p}$. Thus

$$
y=u_{2}+\Phi\left(t^{\prime}\right)
$$

$$
x+y=\left(u_{1}+u_{2}\right)+\Phi\left(t+t^{\prime}\right)
$$

and $x+y \in U_{n}+\Phi\left(A_{m}\right)$. Hence $x+y \in V_{n}$.

Now

$$
\begin{aligned}
\bigcap_{n=1}^{\infty} V_{n} & =\bigcap_{m=2}^{\infty} \bigcap_{n=1}^{\infty}\left(U_{n}+\Phi\left(A_{m}\right)\right) \\
& \left.=\bigcap_{m=2}^{\infty} \overline{\Phi\left(A_{m}\right)} \quad \text { (closure in } \tau\right) \\
& \subset \overline{\Phi\left(A_{2}\right) .}
\end{aligned}
$$

However $\bigcap_{n=1}^{\infty} V_{n}$ is a linear subspace of $E$, and, as $\Phi$ is continuous, $\overline{\Phi\left(A_{2}\right)}$ is bounded. Therefore

and $\gamma(\Phi)$ is Hausdorff.

$$
\bigcap_{n=1}^{\infty} V_{n}=\{0\}
$$

Lemma 2.2. If $\left\{\Phi\left(e_{n}\right): n \in \mathbb{N}\right\}$ is not a $\gamma(\Phi)$-precompact set, then for some infinite subset $M \subset \mathbb{N}, \Phi: c_{0}(M) \rightarrow(E, \tau)$ is an isomorphism on to its image.

Proof. We may find $k \in \mathbb{N}$ such that for any $\gamma(\Phi)$-precompact subset $S$ of $E$, $S+V_{k}$ does not contain $\left\{\Phi\left(e_{n}\right): n \in \mathbb{N}\right\}$. We then select by induction an increasing sequence of integers $p(n)$ such that for every $n$

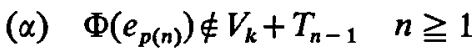

$$
\begin{aligned}
& \text { (B) } \Phi\left(e_{p(n)}\right) \notin U_{k+1}+T_{n-1}+\Phi\left(A_{p(n+1)}\right) \quad n \geqq 1
\end{aligned}
$$

where $T_{0}=\{0\}$ and for $n \geqq 1$

$$
T_{n}=\left\{\sum_{i=1}^{n} a_{i} \Phi\left(e_{p(i)}\right):\left|a_{i}\right| \leqq 1\right\}
$$


Pick $p(1)$ so that $(\alpha)$ holds. Now suppose $p(1) \ldots p(r)$ have been selected so that $(\alpha)$ holds for $1 \leqq n \leqq r$ and $(\beta)$ holds for $1 \leqq n \leqq r-1$. Then by $(\alpha)$

$$
\Phi\left(e_{p(r)}\right) \notin V_{k}+T_{r-1} \text {. }
$$

Since $T_{r-1}$ is $\tau$-compact and symmetric we may have a finite symmetric subset $\Sigma_{r-1}$ of $T_{r-1}$ such that

$$
T_{r-1} \subset \Sigma_{r-1}+U_{k+1} \text {. }
$$

Now $\Phi\left(e_{p(r)}\right) \notin V_{k}+\Sigma_{r-1}$, and hence, for each $\sigma \in \Sigma_{r-1}$ there is a $q(\sigma)$ such that

$$
\Phi\left(e_{p(r)}\right) \notin \sigma+U_{k}+\Phi\left(A_{q(\sigma)}\right) \text {. }
$$

Thus there is a $q=\max \left(q(\sigma): \sigma \in \Sigma_{r-1}\right)$ such that

$$
\Phi\left(e_{p(r)}\right) \notin \Sigma_{r-1}+U_{k}+\Phi\left(A_{q}\right) \text {. }
$$

Since $T_{r-1} \subset \Sigma_{r-1}+U_{k+1}$ we conclude that

$$
\Phi\left(e_{p(r)}\right) \notin T_{r-1}+U_{k+1}+\Phi\left(A_{q}\right) \text {. }
$$

Now pick $p(r+1)>\max (p(r), q)$ to satisfy $(\alpha)$, using the fact that $T_{r}$ is $\gamma(\Phi)$-compact. This completes the inductive construction.

Suppose $\left(a_{i}, \ldots, a_{n}\right)$ is a sequence with $\max _{i}\left|a_{i}\right|=\left|a_{j}\right|=1$. Then

$$
a_{j} \Phi\left(e_{p(j)}\right)=\sum_{i=1}^{n} a_{i} \Phi\left(e_{p(i)}\right)-\sum_{i=1}^{j-1} a_{i} \Phi\left(e_{p(i)}\right)-\sum_{=j+1}^{n} a_{i} \Phi\left(e_{p(i)}\right)
$$

(a summation over the empty set is taken to be zero), and therefore

Hence by $(\beta)$

$$
a_{j} \Phi\left(e_{p(j)}\right) \in \sum_{i=1}^{n} a_{i} \Phi\left(e_{p(i)}\right)+T_{j-1}+\Phi\left(A_{p(j+1)}\right) \text {. }
$$

$$
\sum_{i=1}^{n} a_{i} \Phi\left(e_{p(i)}\right) \notin U_{k+1} \text {. }
$$

Let $M=\{p(1), p(2), \ldots\}$ and consider $\Phi: c_{00} \cap c_{0}(M) \rightarrow(E, \tau)$. If $\Phi\left(t^{(n)}\right) \rightarrow 0$ and $\left\|t^{(n)}\right\|_{\infty} \geqq \varepsilon>0$ for all $n$, then $\Phi\left(\left\|t^{(n)}\right\|_{\infty}^{-1} t^{(n)}\right) \rightarrow 0$. However

$$
\Phi\left(\left\|t^{(n)}\right\|_{\infty}^{-1} t^{(n)}\right) \notin U_{k+1}
$$

for all $n$, and so we have a contradiction. Therefore if $\Phi\left(t^{n}\right) \rightarrow 0$ then

$$
\left\|t^{(n)}\right\|_{\infty} \rightarrow 0
$$

and $\Phi$ is an isomorphism. Clearly $\Phi$ is also an isomorphism on the closure of $c_{00} \cap c_{0}(M)$, i.e. $c_{0}(M)$.

Note that, since $\Phi$ is continuous $\left\{\sum_{n \in k} \phi\left(e_{n}\right): K \subset \mathbb{N}, K\right.$ finite $\}$ is $\gamma(\Phi)$-bounded. Therefore if $\left\{\Phi\left(e_{n}\right): n \in \mathbb{N}\right\}$ is $\gamma(\Phi)$-precompact then $\Phi\left(e_{n}\right) \rightarrow 0$ in $\gamma(\Phi)$.

Theorem 2.3. Suppose $(E, \tau)$ is a topological vector space and $\Phi: c_{0} \rightarrow E$ is a continuous linear map; then either

(i) $\Phi\left(e_{n}\right) \rightarrow 0(\tau)$, 
or

(ii) there is an infinite subset $M$ of $N$ such that $\Phi: c_{0}(M) \rightarrow E$ is an isomorphism onto its image.

Proof. Suppose neither (i) nor (ii) holds. Then we may find a metrisable topological vector space $(F, \mu)$ and a continuous linear map $\Psi: E \rightarrow F$ such that (i) does not hold for $\Psi \Phi$. Then (ii) also must fail for $\Psi \Phi$, and so we may reduce consideration to the metrisable case for $\tau$. We may also suppose that $(E, \tau)$ is complete. As above, let $\left(U_{n}\right)$ be a base of neighbourhoods for $\tau$.

Now by Lemma 2.2, $\Phi\left(e_{n}\right) \rightarrow 0 \gamma(\Phi)$. Let $\bar{\gamma}$ be the finest vector topology such that $\bar{\gamma} \leqq \tau$ and $\Phi\left(e_{n}\right) \rightarrow \mathrm{O}(\bar{\gamma})(\bar{\gamma}$ is given by all $\tau$-continuous $F$-semi-norms which make $\Phi\left(e_{n}\right)$ a null sequence). Then $\bar{\gamma}$ is Hausdorff since $\gamma(\Phi)$ is Hausdorff. Now let $\bar{\gamma}$ be the metrisable topology with a base of neighbourhoods $\left(\bar{U}_{n}\right)$ (closure in $\bar{\gamma})$. Then if $\bar{\gamma}=\bar{\gamma}$ the identity map in $i:(E, \bar{\gamma}) \rightarrow(E, \tau)$ is almost continuous and therefore by the Closed Graph Theorem (Kelley (9), p. 213), $\bar{\gamma}=\tau$. Since we are assuming (i) to be false we conclude that $\bar{\gamma}<\bar{\gamma} \leqq \tau$. Therefore

$$
\Phi\left(e_{n}\right) \nrightarrow 0(\bar{\gamma})
$$

and so by Theorem 3.2 of (8), there is a subsequence $\left(\Phi\left(e_{n}\right): n \in M\right)$ which is a regular basic sequence in $(E, \tilde{\gamma})$. (A sequence is regular if it is bounded away from zero and basic if it forms a basis for its closed linear span in the completion of $(E, \bar{\gamma})$.)

Now if $t \in c_{0}(M)$ then $\Sigma t_{n} \Phi\left(e_{n}\right)$ converges in $(E, \tau)$ and hence in $(E, \bar{\gamma})$. Then as $\left(\Phi\left(e_{n}\right): n \in M\right)$ is $\bar{\gamma}$-regular it is equivalent to the unit vector basis of $c_{0}$. By a result of Arsove and Edwards (1), $\Phi: c_{0}(M) \rightarrow G$ is an isomorphism where $G$ is the closed linear span of $\Phi\left(e_{n}\right)$ in $(E, \bar{\gamma})$. Then $G$ is also closed in $(E, \tau)$ and by the Open Mapping Theorem $\Phi$ is also an isomorphism for the topology $\tau$. This contradicts our assumption that (ii) was false.

Theorem 2.4. Let $(E, \tau)$ be a topological vector space containing no copy of $c_{0}$ Then any bounded linear map $\Phi: c_{0} \rightarrow E$ takes the unit ball $B$ of $c_{0}$ into a precompact subset of $E$.

Proof. If $\Phi(B)$ is not precompact, we may find a neighbourhood $U$ of zero in $E$ and a sequence $t^{(n)}$ in $c_{00} \cap B$ such that $\Phi\left(t^{(n)}\right)-\Phi\left(t^{(m)}\right) \notin U$ for $n \neq m$.

By selecting a subsequence we may suppose $\left(t^{(n)}\right)$ is co-ordinatewise convergent in $l_{\infty}$. Thus $t^{(n)}-t^{(n+1)} \rightarrow 0$ co-ordinatewise. We may then select a subsequence $s^{(n)}$ of $\left(t^{(n)}-t^{(n+1)}\right)$ which is disjoint (i.e. if $n \neq m s_{k}^{(n)} \cdot s_{k}^{(m)}=0$ for all $k$ ). Define $\Psi: c_{0} \rightarrow E$ by

$$
\Psi(u)=\sum_{i=1}^{\infty} u_{i} \Psi\left(s^{(i)}\right) .
$$

As $\Psi\left(s^{(n)}\right) \notin U$, we may conclude from Theorem 2.3 that $E$ contains a subspace isomorphic to $c_{0}$.

If $E$ is complete then the hypotheses of Theorem 2.4 ensure that $\sum_{n=1}^{\infty} \Phi\left(e_{n}\right)$ converges. 


\section{Operators on $l_{\infty}$}

Lemma 3.1. Let $E$ be a separable metrisable topological vector space and suppose $\Phi: l_{\infty} \rightarrow E$ is a continuous operator such that $\Phi\left(c_{0}\right)=0$. Then there is an infinite subset $M$ of $N$ such that $\Phi\left(l_{\infty}(M)\right)=0$.

Here $l_{\infty}(M)=\left\{t \in l_{\infty}: t_{i}=0, i \notin M\right\}$.

Proof. We may assume that $E$ is complete. Let $\left(M_{\alpha}: \alpha \in \mathscr{A}\right)$ be an uncountable collection of infinite subsets of $\mathbb{N}$ such that $M_{\alpha} \cap M_{\beta}$ is finite for each $\alpha \neq \beta$, see (19). Suppose if possible that for each $\alpha \in \mathscr{A}$ there exists $t^{(\alpha)} \in l_{\infty}\left(M_{\alpha}\right)$ with $\left\|t^{(\alpha)}\right\|_{\infty}=1$ and $\Phi\left(t^{(x)}\right) \neq 0$. Let $\mathscr{A}_{k}=\left\{\alpha: \Phi\left(t^{(\alpha)}\right) \notin V_{k}\right\}$ where $\left(V_{k}\right)$ is a base of neighbourhoods of 0 in $E$. Then for some $k, \mathscr{A}_{k}$ is uncountable; however $\left(\Phi\left(t^{(z)}\right): \alpha \in \mathscr{A}_{k}\right)$ is separable and hence there is a sequence $\left(\alpha_{n}\right)$ in $\mathscr{A}_{k}$ such that

where $x_{0} \neq 0$. Then for any $p$

$$
\Phi\left(t^{\left(\alpha_{n}\right)}\right) \rightarrow x_{0},
$$

$$
\Phi\left(\sum_{n+1}^{n+p} t^{\left(x_{i}\right)}\right) \rightarrow p x_{0} .
$$

However since $M_{\alpha_{i}} \cap M_{\alpha_{j}}$ is finite if $i \neq j$ and $\Phi\left(c_{0}\right)=0$ we conclude that

$$
\Phi\left(\sum_{n+1}^{n+p} t^{\left(\alpha_{l}\right)}\right) \in \Phi(B),
$$

where $B$ is the unit ball of $l_{\infty}$. Thus $p x_{0} \in \overline{\Phi(B)}$ for any $p$ and we have a contradiction.

Theorem 3.2. Let $(E, \tau)$ be a separable topological vector space, and let $\Phi: l_{\infty} \rightarrow E$ be a continuous linear operator. Then $\Phi\left(e_{n}\right) \rightarrow 0$.

Proof. Since $E$ may be embedded in a product of separable metrisable spaces, it is sufficient to assume that $(E, \tau)$ is metrisable and complete. Now suppose $\Phi\left(e_{n}\right) \rightarrow 0$ in $(E, \tau)$. Then there is an infinite subset $M$ of $N$ such that $\Phi: c_{0}(M) \rightarrow(E, \tau)$ is an isomorphism onto a closed subspace $G$ of $E$.

Let $\pi: E \rightarrow E / G$ be the quotient map; then $\pi \Phi=0$ on $c_{0}(M)$ and by Lemma 3.1 there is an infinite subset $M_{0}$ of $M$ such that $\pi \Phi=0$ on $l_{\infty}\left(M_{0}\right)$, i.e. $\Phi\left(l_{\infty}\left(M_{0}\right)\right) \subset G$. Now as $G \cong c_{0}$, we may apply the theorem of Grothendieck (6, p. 173), or Rosenthal (16) to deduce $\Phi$ is weakly compact on $l_{\infty}\left(M_{0}\right)$ and hence $\sum_{n \in M_{0}} \Phi\left(e_{n}\right)$ is weakly subseries convergent in $G$. By the Orlicz-Pettis Theorem $\Phi\left(e_{n}\right) \rightarrow 0$ (see e.g. (5) p. 318 , (12) or (15)).

It is very possible Theorem 3.2 can be extended to topological vector spaces containing no copy of $l_{\infty}$. However, here we have only a partial result. The technique of the following theorem is essentially found in Drewnowski (4). We identify $l_{\infty}$ as $C(\beta N)$ and thus we can define exhaustive operators as in the introduction.

Theorem 3.3. Let $\Phi: l_{\infty} \rightarrow(E, \tau)$ be a continuous linear operator, and suppose there is a Hausdorff vector topology $\rho$ on $E$ such (i) $\Phi: l_{\infty} \rightarrow(E, \rho)$ is exhaustive (ii) 
$\tau$ is $\rho$-polar, i.e. has a base of $\rho$-closed neighbourhoods of 0 . Then if $\Phi\left(e_{n}\right) \rightarrow 0$ in $\tau$, there is an infinite subset $M$ of $\mathbb{N}$ such that $\Phi: l_{\infty}(M) \rightarrow(E, \tau)$ is an isomorphism onto its image.

Proof. By (8) Proposition 2.1, there is a $\tau$-continuous $F$-semi-norm $\eta$ of the form

$$
\eta(x)=\sup (\lambda(x): \lambda \in \Lambda)
$$

where $\Lambda$ is a collection of $\rho$-continuous $F$-semi-norms and such that for an infinite subset $M_{0}$ of $\mathbb{N}$

$$
\eta\left(\Phi\left(e_{n}\right)\right) \geqq 1 \quad n \in M_{0} .
$$

By Theorem 2.3 we may suppose that for some subsequence $M_{1}$ of $M_{0}$, $\Phi: c_{0}\left(M_{1}\right) \rightarrow\left(\hat{E}, \eta_{1}\right)$ is an embedding (where $\left(\hat{E}, \eta_{1}\right)$ is the Hausdorff quotient of $(E, \eta))$. Thus if $t \in c_{0}\left(M_{1}\right),\|t\|_{\infty}=1$ then

$$
\eta(\Phi(t)) \geqq \theta>0 \text {. }
$$

We next select a sequence $\left(m_{k}: k=1,2, \ldots\right)$ in $\mathbb{N}$ and a sequence

$$
\left(M_{k}: k=1,2, \ldots\right)
$$

of infinite subsets of $\mathbb{N}$ by induction. First choose $m_{1} \in M_{1}$. Next given $\left(m_{1}, \ldots, m_{k}\right)$ and $\left(M_{1}, \ldots, M_{k}\right)$ let $S_{k}$ be a finite subset of

$$
L_{k}=\left\{\sum_{i=1}^{k} t_{i} \Phi\left(e_{m_{i}}\right): \max \left|t_{i}\right|=1\right\}
$$

such that for $x \in L_{k}$ there exists $s \in S_{k}$ with

$$
\eta(x-s) \leqq \frac{1}{8} \theta .
$$

For each $s \in S_{k}$ pick $\lambda_{s} \in \Lambda$ such that

$$
\lambda_{s}(s) \geqq \eta(s)-\frac{1}{8} \theta .
$$

Now let $M_{k}=\bigcup_{n}^{\infty} P_{n}$ where $\left(P_{n}\right)$ is any sequence of disjoint infinite sets. Since $\Phi$ is exhaustive for $\rho$ we may find $n_{0}$ such that for $t \in l_{\infty}\left(P_{n_{0}}\right),\|t\|_{\infty} \leqq 1$ and $s \in S_{k}$

$$
\lambda_{s}(\Phi(t)) \leqq \frac{1}{8} \theta .
$$

Let $M_{k+1}=P_{n_{0}}$ and then choose $m_{k+1} \in M_{k+1}$. This constructs a set

$$
M=\left(m_{1}, m_{2}, \ldots, m_{k}, \ldots\right)
$$

such that $\left(m_{k+1}, m_{k+2}, \ldots\right) \subset M_{k+1}$ for all $k$.

Now suppose $t \in l_{\infty}(M)$ with $\|t\|_{\infty}=1$. For $\varepsilon>0$ there exists $k$ such that $\left|t_{m_{k}}\right|>1-\varepsilon$; thus there exists $\delta$ with $|\delta|<\varepsilon$ and such that if $t^{\prime}=t+\delta e_{m_{k}}$ then $\left\|t^{\prime}\right\|=\left|t_{m_{k}}^{\prime}\right|=1$. Then let $t^{\prime \prime}=\sum_{i=1}^{k} t_{m_{i}}^{\prime} e_{m_{i}}$ and choose $s \in S_{k}$ such that

$$
\eta\left(\Phi\left(t^{\prime \prime}\right)-s\right) \leqq \theta \frac{1}{8} \text {. }
$$


Then

Hence

$$
\begin{aligned}
\eta\left(\Phi\left(t^{\prime}\right)\right) & \geqq \lambda_{s}\left(\Phi\left(t^{\prime}\right)\right) \\
& \geqq \lambda_{s}\left(\Phi\left(t^{\prime \prime}\right)\right)-\frac{1}{8} \theta \\
& \geqq \lambda_{s}(s)-\frac{1}{4} \theta \\
& \geqq \eta(s)-\frac{3}{8} \theta \\
& \geqq \frac{5}{8} \theta .
\end{aligned}
$$

$$
\eta(\Phi(t)) \geqq \frac{5}{8} \theta-\eta\left(\varepsilon \Phi\left(e_{m_{k}}\right)\right),
$$

and therefore, as $\varepsilon>0$ is arbitrary and $\left(\Phi\left(e_{n}\right): n \in \mathbb{N}\right)$ is bounded,

$$
\eta(\Phi(t)) \geqq \frac{s}{8} \theta .
$$

It follows easily that $\Phi$ is an isomorphism on $l_{\infty}(M)$.

\section{Applications}

In this section we collect together the main results of the paper, which are deductions from the more technical results of Sections 2 and 3.

Theorem 4.1. Let $E$ be a topological vector space containing no copy of $c_{0}$; then every continuous linear operator $\Phi: C(S) \rightarrow E$, where $S$ is compact Hausdorff, is exhaustive (and can therefore be represented in the form

$$
\Phi(f)=\int_{s} f(s) d \mu
$$

where $\mu$ is a regular countably additive E-valued vector measure defined on the Borel sets of $S$ ).

Proof. Let $\left(f_{n}\right)$ be any sequence of positive functions in $C(S)$ such that

Then we can define $\Psi: c_{0} \rightarrow E$ by

$$
\sup _{s} \sum_{n=1}^{\infty} f_{n}(s)<\infty \text {. }
$$

$$
\Psi(t)=\Phi\left(\sum_{n=1}^{\infty} t_{n} f_{n}\right)
$$

$\left(\sum_{n=1}^{\infty} t_{n} f_{n}\right.$ converges in the norm topology of $\left.C(S)\right)$. By Theorem $2.3 \Psi\left(e_{n}\right) \rightarrow 0$ i.e. $\Phi\left(f_{n}\right) \rightarrow 0$ and so $\Phi$ is exhaustive.

Theorem 4.2. Suppose $S$ is a $\sigma$-Stonian compact Hausdorff space and that $E$ is a separable topological vector space. Then any continuous linear operator $\Phi: C(S) \rightarrow E$ is exhaustive.

Proof. Suppose $f_{n} \in C(S), f_{n} \geqq 0$ and

E.M.S. $-19 / 3-U$

$$
\sup _{s} \sum_{n=1}^{\infty} f_{n}(s)<\infty
$$


Then since $C(S)$ is $\sigma$-order-complete we can define for $t \in l_{\infty}$ and $t \geqq 0$ the order-sum $o-\sum_{n=1}^{\infty} t_{n} f_{n}=\sup _{n} \sum_{k=1}^{n} t_{k} f_{k}$. We can extend this definition to a linear map $\Gamma: l_{\infty} \rightarrow C(S)$ and $\Gamma$ is continuous. Now let $\Psi=\Gamma \Phi$ and apply Theorem 3.2.

Theorem 4.3. Suppose $(E, \tau)$ is an F-space containing no copy of $l_{\infty}$ and $\Phi: C(S) \rightarrow E$ is a continuous linear operator which is exhaustive for a weaker Hausdorff vector topology $\rho$ on $E$. Then $\Phi$ is exhaustive for $\tau$.

Proof. Let $\gamma$ be the largest vector topology on $E$ such that $\gamma \leqq \tau$ and $\Phi$ is $\gamma$-exhaustive. Let $\bar{\gamma}$ be the topology with a base of $\tau$-neighbourhoods consisting of the $\gamma$-closures of $\tau$-neighbourhoods of 0 .

Suppose now for some $f_{n} \geqq 0$ with sup $\Sigma f_{n}(s)<\infty$ that $\Phi\left(f_{n}\right) \rightarrow 0(\bar{\gamma})$. Then we may form the map $\Psi: l_{\infty} \rightarrow E$ as in Theorem 4.2 and by Theorem 3.3 there is infinite subset $M$ of $\mathbb{N}$ such that $\Psi: l_{\infty}(M) \rightarrow(E, \bar{\gamma})$ is an embedding. Hence $\Psi: l_{\infty}(M) \rightarrow(E, \tau)$ is an embedding and this contradicts the hypotheses of the theorem. Hence $\Phi\left(f_{n}\right) \rightarrow 0(\bar{\gamma})$ and so $\Phi$ is $\bar{\gamma}$-exhaustive. However, $\bar{\gamma} \leqq \tau$ and therefore $\bar{\gamma} \leqq \gamma$; thus the identity $I:(E, \gamma) \rightarrow(E, \tau)$ is almost continuous and by the Closed Graph Theorem (Kelley (9), p. 213) is also continuous, i.e. $\gamma=\tau$ and $\Phi$ is $\tau$-exhaustive.

Remark. If $E$ has the property that the continuous linear operators with separable range separate points then a topology $\rho$ can also be found to satisfy the conditions of Theorem 4.3.

Next we mention two other applications. Our first result generalises a theorem of Diestel (3).

Theorem 4.4. Let $E$ be a separable locally bounded $F$-space, and let $\mathscr{S}$ be a $\sigma$-algebra of subsets of a set $S$. Let $\mu: \mathscr{S} \rightarrow E$ be a bounded (finitely-additive) measure. Then $\mu$ is exhaustive.

Note. A measure $\mu$ is called exhaustive or strongly bounded if for any sequence $\left(S_{n}\right)$ of disjoint sets $\mu\left(S_{n}\right) \rightarrow 0$.

Proof. Since $E$ is locally bounded, the topology may be given by a $p$-norm $\|$.$\| where 0<p \leqq 1$. Let

$$
\sup _{S \in \mathscr{Y}}\|\mu(S)\|=\theta \text {. }
$$

We use a technique due to Robertson (15); $\mu$ may be extended to a linear map $\Phi_{0}$ on the simple functions $\Sigma(\mathscr{S})$ on $\mathscr{S}$. Then $\Sigma(\mathscr{S})$ is a normed space under

suppose $f \in \Sigma(\mathscr{S})$ and $\|f\|_{\infty} \leqq 1$. Then

$$
\|f\|=\sup _{s \in S}|f(s)|
$$

$$
\Phi_{0}(f)=\sum_{=1}^{\infty} 2^{-i}\left(\mu\left(S_{i}\right)-\mu\left(T_{i}\right)\right)
$$


where $S_{i}, T_{i} \in \mathscr{S}$ (only finitely many of $S_{i}, T_{i}$ are distinct). Then

$$
\left\|\Phi_{0}(f)\right\| \leqq 2 \theta \sum_{i=1}^{\infty} 2^{-i p} \leqq \frac{\theta 2^{1+p}}{1-2^{-p}}
$$

Thus $\Phi_{0}: \Sigma(\mathscr{S}) \rightarrow E$ is continuous and extends to a continuous operator $\Phi: B(\mathscr{S}) \rightarrow E$ where $B(\mathscr{S})$ in the space of bounded $\mathscr{S}$-measurable functions on $S$. Using the techniques of Theorem 4.2 it follows that if $\left(S_{n}\right)$ is a disjoint sequence in $\mathscr{S}, \mu\left(S_{n}\right)=\Phi\left(\chi_{s_{n}}\right) \rightarrow 0$ where $\chi_{s_{n}}$ is the characteristic of $S_{n}$. (Alternatively $B(\mathscr{S})$ is isometrically isomorphic to $C(T)$ where $T$ is $\sigma$-Stonian.)

Clearly the preceding theorem generalises to semi-convex topological vector spaces (i.e. spaces which can be embedded in a product of locally bounded spaces).

A $C$-series is a sequence $x_{n}$ in a topological vector space such that $\Sigma t_{n} x_{n}$ converges whenever $t_{n} \rightarrow 0$. If $E$ is a space such that every $C$-series converges, then $E$ is called a $C$-space (Schwartz (17), Thomas (18)). Clearly Theorem 2.3 yields

Theorem 4.4. A complete topological vector space is a $C$-space if and only if it contains no subspace isomorphic to $c_{0}$.

A topological vector space is said to have property $(O)$ (Orlicz (13), Labuda (10)) if every series $\Sigma x_{n}$ in $E$ such that the set $\left\{\sum_{n \in \Delta} x_{n}: \Delta \subset \mathrm{N}, \Delta\right.$ finite $\}$ is bounded, is also convergent. Again by Theorem 2.3 and a similar argument to Theorem 4.3 we conclude

Theorem 4.5. A complete semi-convex topological vector space $E$ has property $(O)$ if and only if $E$ contains no subspace isomorphic to $c_{0}$.

\section{REFERENCES}

(1) M. G. Arsove and R. E. Edwards, Generalised bases in topological linear spaces, Studia Math. 19 (1960), 95-113.

(2) C. Bessaga and A. PelczyŃski, On bases and unconditional convergence of series in Banach spaces, Studia Math. 17 (1958), 151-164.

(3) J. Diestel, Applications of bases and weak compactness to vector measures and vectorial integration, Rev. Roum. Math. Pures et Appl. 18 (1973), 211-224.

(4) L. DrewnowskI, Another note on Kalton's theorem (to appear).

(5) N. Dunford and J. T. SchwarTZ, Linear Operators, Vol. I (Interscience, New York, 1958).

(6) A. GRotheNDiecK, Sur les applications linéaires faiblement compactes d'espaces du type $\mathrm{C}(K)$, Canad. J. Math. 5 (1953), 129-173.

(7) N. J. Kal.ron, Topologies on Riesz groups and applications to measure theory, Proc. London Math. Soc. (3) 18 (1974), 253-273. 
(8) N. J. Kalton, Basic sequences in F-spaces and their applications, Proc. Edinburgh Math. Soc. 19 (1974), 151-167.

(9) J. L. KelleY, General Topology (van Nostrand, New York, 1955).

(10) G. KöTHE, Topological Vector Spaces (Springer, Berlin, 1969).

(11) I. Labuda, Sur quelques théorèmes du type d'Orlicz-Pettis I, Bull. Acad. Polon. Sci. 21 (1973), 127-132.

(12) C. W. McArthur, On a theorem of Orlicz and Pettis, Pacific J. Math. 22 (1967), 297-302.

(13) W. ORLICz, Absolute continuity of set functions with respect to a finitely subadditive measure, Prace Mat. 14 (1970), 101-118.

(14) A. PelczyŃski, Banach spaces on which every unconditionally converging operator is weakly compact, Bull. Acad. Polon. Sci. 10 (1962), 641-648.

(15) A. P. Robertson, On unconditional convergence in topological vector spaces, Proc. Roy. Soc. Edinburgh, Sect. A 68 (1969), 145-157.

(16) H. P. Rosenthal, On relatively disjoint families of measures, with some applications to Banach space theory. Studia Math. 37 (1971), 13-36.

(17) L. SchwaRTZ, Un théorème de la convergence dans les espaces $L^{p}, 0 \leqq p<\infty$, C.R. Acad. Sci. Paris, Ser. A 268 (1969), 704-706.

(18) E. Thomas, Sur le théorème d'Orlicz et un problème de M. Laurent Schwartz, C.R. Acad. Sci. Paris, Ser. A. 267 (1968), 7-10.

(19) R. J. WhITLEY, Projecting $m$ onto $c_{0}$, Amer. Math. Monthly 73 (1966), 285-286.

UNIVERSITY COLlege of SWANSEA

SINGLETON PARK

SWANSEA SA2 8PP 